\title{
Cellular Changes Induced by Caffeine in Developing Tracheary Elements
}

\author{
J.S. Sadick, J.M. Sumner, P.C. Lovejoy, and T.P. Owen, Jr. \\ Department of Botany, Connecticut College, 270 Mohegan Avenue, New London, CT 06320
}

Tracheary elements, the tissue through which water transport occurs in plants, is a multistep process in which microtubules realign, Golgi vesicles deliver carbohydrates and proteins to the plasma membrane for directed wall deposition, and ultimately apoptosis occurs [1]. These various cellular events are coordinated by precise changes in gene expression mediated by hormonal control [2]. As this process is difficult to observe or manipulate within the plant body, a cell culture system is frequently used [3] where mechanically isolated leaf mesophyll cells from Zinnia elegans L. are placed into an aseptic tissue culture with a high auxin to cytokinin hormone ratio. Approximately $60 \mathrm{~h}$ later, a population of cells begins to show secondary cell walls with a cell wall pattern similar to that of xylary cells in vivo (Figs. 1-3). In contrast, cells incubated in a media with basal hormones do not show this differentiation.

We have shown [4] that disruption of endomembrane traffic from the rER to the Golgi apparatus, using the inhibitor brefeldin A, can be followed by fluorescent and transmission electron microscopy in cultures of Zinnia tracheary elements. For the current study, we are examining the effects of caffeine on tracheary element differentiation with a particular interest in Golgi vesicle traffic and cell cycle regulation. Although considerable research has been performed to examine the effects of caffeine in mammalian cellular systems, particularly on G-protein calcium ion pathways in vesicle formation as well as regulation of the cell cycle, the study of these subjects collectively has not been carefully examined in plant cells.

When $1 \mathrm{mM}$ of caffeine is added $36 \mathrm{~h}$ after starting either an inductive or noninductive mesophyll culture, a population of multinucleate cells, with the number of nuclei ranging from bi- to pentanucleate, formed by $72 \mathrm{~h}$ (Fig. 4). This caffeine concentration induced the highest number of multinucleate cells without causing cell death. Additionally, this concentration of caffeine significantly diminished the formation of cells with secondary wall growths $(p=0.00)$. There was no significant different $(p=0.057)$ in the formation of the multinucleate cells between the two types of media. Although many species of plants are normally multinucleate, Zinnia elegans are not. Specifically, the differentiation of the mesophyll cells into tracheary elements occurs without intervening mitosis [5]. The number of nuclei was observed by staining with DAPI, a fluorescent dye that preferentially binds to DNA (Fig. 5). Cell wall formation was tracked with Fluorescent Brightner 28 (FB-28; Sigma Chemical Co.) which binds to cellulose. FB-28 staining or DIC microscopy did not reveal the presence of cell wall plates between the nuclei of multinucleated cells; we are currently examining these cells by TEM to verify an entirely cellular state. We will also survey the morphology of the Golgi and associated vesicles for changes due to the caffeine incubation (Figs. 6-8).

In the coming months, the project will expand to examine the potential similarities or differences between developed multinucleate plant cells versus multinucleate mammalian tumor cells as many cancerous tumor cells are also multinucleate [6]. Since cytokinesis can be interrupted due to failure of microtubule function in mammalian cells leading to a multinucleate state [7], could the addition 
of caffeine to the Zinnia cultures have a similar response? It is possible that a malfunctioning cytoskeleton could affect both the plant's cell cycle as well as the formation of a secondary cell wall, since Golgi vesicles are transported to the plasma membrane by microtubules. Future data will be obtained to further support past hypotheses on caffeine's intramolecular effect or to raise new ideas on the mechanism by which caffeine acts upon the cell [8].

\section{References}

[1] H. Höfte, Curr. Biol. 20 (2010) R450.

[2] S. Turner et al., Ann. Rev. Plant Biol. 58 (2007) 407.

[3] H. Fukuda and A. Komamine, Plant Physiol. 65 (1980) 57.

[4] M. Rojas et al., Int. J. Plant Sci. 160 (1999) 683.

[5] H. Fukuda and A. Komamine, Plant Physiol. 65 (1980) 61.

[6] E. Marrazzo et al., Eur. J. Cancer 45 (2009) 443.

[7] C. Caldwell et al., J. Cell Biol. 178 (2007) 1109.

[8] Research supported by Keck Undergraduate Science Fellowships at Connecticut College to J.S. and J.S. The help of Evan Studwell and Aaron Feldman is appreciated. The TEM was supported by NSF to T.P.O.

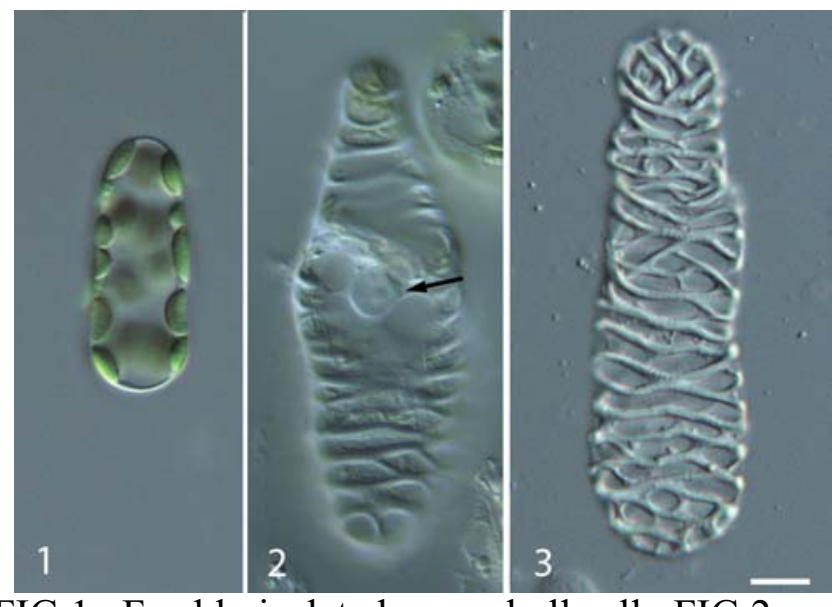

FIG 1. Freshly isolated mesophyll cell. FIG 2.

Differentiating TE with nucleus (arrow). FIG 3. Fully formed TE. Bar $=20 \mu \mathrm{m}$.

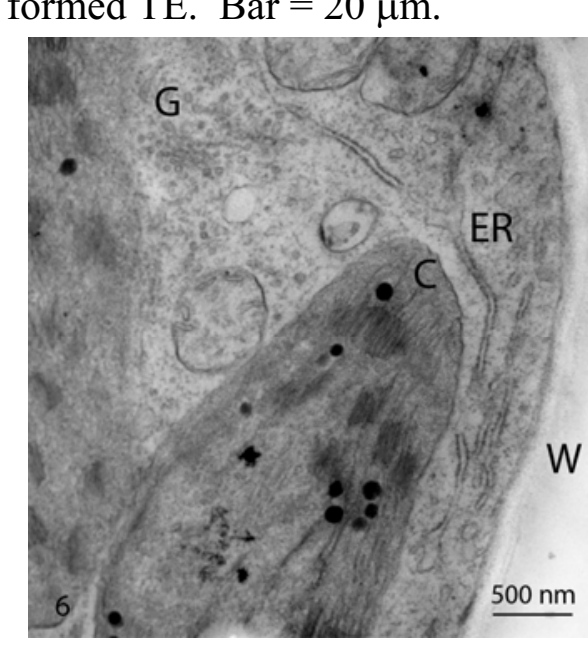

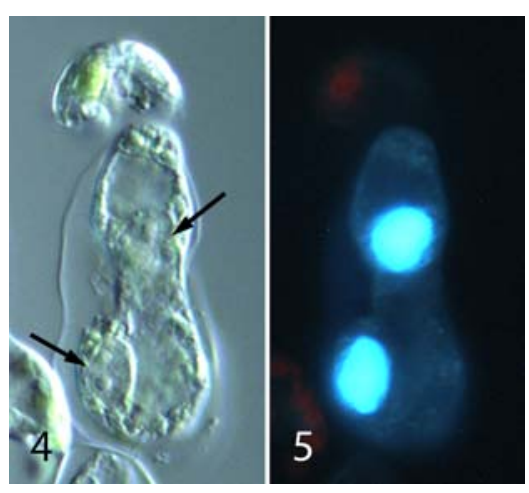

FIG 4. Caffeine TE with two nuclei (arrows). FIG 5. Same cell stained with DAPI for DNA.

FIGS 6-8. TEM of differentiating control TEs with an active endomembrane system $(\mathrm{G}=$ Golgi, $\mathrm{C}=$ chloroplast, $\mathrm{CV}=$ coated vesicle, $\mathrm{W}=$ primary wall, $2 \mathrm{~W}=$ secondary wall, arrow $=$ microtubule). 